\title{
Los gestos, una manera de comunicar matemática: el caso particular de las funciones
}

\section{Gestures as a way to communicate mathematics: The particular case of functions}

\section{Luis Sandoval-Troncoso ${ }^{1}$ \\ Carlos Ledezma²}

\begin{abstract}
Resumen: En este artículo se presenta un estudio del abordaje de las funciones, cuyo foco se centró en la actividad gestual, y que se llevó a cabo con estudiantes de una universidad chilena que cursaban la asignatura de Cálculo I. Como referentes teóricos, se consideraron la Teoría de la Objetivación y la Teoría Comognitiva. Los resultados obtenidos, mostraron la relevancia del discurso que se produce mediante los gestos en la actividad colectiva, aportando a la generación y aparición de elementos matemáticos claves del objeto función de variable real, constituyéndose el gesto como motor impulsor del discurso matemático del sistema semiótico de significación cultural. Además, el estudio evidenció como factor importante la ruptura del discurso tradicional de la enseñanza de la matemática a nivel universitario.
\end{abstract}

Palabras clave: actividad gestual, discurso matemático, función de variable real, Teoría Comognitiva, Teoría de la Objetivación.

Fecha de recepción: 09 de enero de 2020. Fecha de aceptación: 02 de marzo de 2021.

1 Departamento de Matemática y Estadística, Universidad de La Frontera, Chile, luis.sandoval@ufrontera.cl, orcid.org/0000-0003-2844-5661

2 Departamento de Educación Lingüística y Literaria y de Didáctica de las Ciencias Experimentales y de la Matemática, Universitat de Barcelona, España, cledezar25@alumnes.ub.edu, orcid.org/0000-0001-9274-7619 


\begin{abstract}
This paper presents a study on the approach to functions, whose focus was on gestural activity, and which was carried out with students from a Chilean university who were studying Calculus I. Theory of Objectivation and Comognitive Theory were considered as theoretical referents. The results obtained showed the relevance of the discourse that is produced through gestures in the collective activity, contributing to the generation and emergence of key mathematical elements of the object function of real variable. In this context, gesture was the driving force behind the mathematical discourse of the semiotic system of cultural significance. In addition, this study made evident the breakdown of the traditional discourse of mathematics teaching at the university level as an important factor.
\end{abstract}

Keywords: gestural activity, mathematical discourse, function of real variable, Comognitive Theory, Theory of Objectivation.

\title{
1. INTRODUCCIÓN
}

Hoy en día, existen variadas herramientas tecnológicas en la matemática que permiten a los estudiantes obtener información en torno a una gráfica y deducir otras, producto de la visualización de estas por medio de un software. Investigaciones al respecto, como las de Artigue (2011), explican la importancia que tiene la tecnología en el aprendizaje matemático de los estudiantes, quienes dan significado y valor a la matemática a través de la interacción con la tecnología. Según Gómez-Chacón (2010), la tecnología permite la generación de ejemplos y representa una ayuda en la elaboración de demostraciones, conectando lo geométrico con lo algebraico. Otros, como Gamboa (2007), indican que su aporte en la matemática va en dirección a que el estudiante justifique sus respuestas de una manera más formal.

Sin embargo, además de la tecnología, existen otros factores que también generarían oportunidades de aprendizaje de la matemática, especialmente a nivel universitario, cuya enseñanza se centra principalmente en el álgebra. Es por ello que esta propuesta permite romper este esquema con las oportunidades que podría dar el uso de la corporalidad como un nuevo ambiente de trabajo matemático en el aula, lo que estaría relacionado con lo propuesto por Radford (2006a, 2010), y que contempla acciones como los gestos, el lenguaje y los 
símbolos. Ejemplo de ello se encuentra en Arzarello et al., (2009), quienes amplían los enfoques semióticos clásicos a través de lo que denominan paquete semiótico y haz semiótico que, junto a la multimodalidad, permiten a los estudiantes lograr comprender conceptos matemáticos de manera más efectiva. Dicha comprensión es alcanzada por los sujetos bajo este enfoque multimodal referido a gestos, concluyendo así que dichos factores "ayudan a cerrar la brecha entre la experiencia mundana y la matemática más formal" (Arzarello et al., 2009, p. 98). Los autores también indican que los gestos tienen una función comunicativa, permitiendo formas alternativas de incorporar y organizar información que el estudiante no puede manifestar de manera verbal o formal.

\subsection{ANTECEDENTES}

Es destacable que, esta forma de enfocar el conocimiento, ha sido abordada desde otros contextos: en lo lingüístico, lo psicológico e, inclusive, en la comunicación cotidiana (e.g. Goldin-Meadow, 2003; Kita, 2000; McNeill, 1992; 2005), y que de a poco ha sido introducida en el ambiente del aprendizaje de la matemática, donde ha evidenciado resultados positivos. Al respecto, estudios como el de Radford et al., (2009), indican que, en los actos de conocimiento de los individuos, las modalidades sensoriales -es decir, lo táctil, lo perceptivo, lo kinestésico-, se convierten en partes integrales de los procesos cognitivos que estos desarrollan, lo que implica que la matemática es un producto poderoso y estable del ser humano.

Continuando en esta línea, existen trabajos que enfatizan las acciones corporales como una actividad clave en el aprendizaje de la matemática. Soto-Andrade (2018), por ejemplo, se refiere a que "la metáfora y la ejecución (en el sentido de actuar físicamente) desempeñan un papel clave en el aprendizaje de las matemáticas" (p. 634), cuestión que pudo concluir en una actividad realizada con matemáticos, estudiantes y profesores de matemática en una universidad chilena. En cuanto a lo enactivo, Varela, Thompson y Rosch (1991) indican que, en esta concepción, las duplas conocedor y conocido, y organismo y medio ambiente, coevolucionan en un proceso constante de llegar a ser. En esta perspectiva, los aportes de lo enactivo permitirían un aprendizaje de la matemática de modo más dinámico, haciendo de la corporalidad una herramienta potente para comunicar propiedades y/o atributos de un objeto matemático. Este hecho, hace una diferencia con la matemática tradicional de lápiz y papel, 
demostrando así esa coevolución a la que aluden Varela et al. (1991). Una posibilidad que traería consigo esta concepción enactivista del aprendizaje, es la perdurabilidad del conocimiento en el estudiante a través del tiempo. Las acciones corporales pretenden ser un impulsor natural de un discurso que trasciende de lo visual o lo simbólico-algebraico, para construir un nuevo discurso matemático.

En concordancia a lo planteado anteriormente, se encuentran estudios que señalan que, el gesto en matemáticas y en otras formas de conocimiento, son "inseparables de las herramientas simbólicas [y esa cognición es] un fenómeno de forma cultural" (Sfard y McClain, 2002, p. 156), afirmación que abre el camino hacia lo que Radford (2006b) menciona como sistema semiótico de significación cultural (el cual será abordado más adelante), señalando que el gesto, como un signo, juega un rol protagónico en la formación de patrones en el álgebra. Así lo demuestran los trabajos de Radford (2010) llevados a cabo con estudiantes de primaria en 2009, en que la actividad con las manos, en especial de los dedos, son de gran ayuda en el aprendizaje del álgebra temprana. En cuanto a esta última cuestión, estudios como el de McNeill (1992) clasifican los gestos, dependiendo de la funcionalidad que estos cumplen, en icónicos, rítmicos, metafóricos o deícticos. En esta misma línea, Alibali et al., 2019) sostienen que los maestros usan sus propios gestos para resaltar y dejar sin ambigüedades las declaraciones de los estudiantes mientras discuten una tarea, asegurando que la actividad gestual conecta el discurso de los sujetos con el contexto, permitiendo que dicho discurso sea adoptado con precisión.

Por otra parte, existen trabajos (Mancilla y Puentes, 2015; Suárez y Pietrosemolli, 2007) que, basándose en la clasificación de McNeill (1992), han abordado el tema de los gestos en contextos lingüísticos, concluyendo que, una de las cuestiones relevantes de la clasificación de gestos dentro de la acción comunicativa, es la de "distinguir las funciones que estos tienen cuando están acompañando la producción oral” (Suárez y Pietrosemolli, 2007, p. 80). Esta afirmación es una base para conseguir los objetivos de este estudio, pues se pretende evidenciar esta conexión entre gestos y habla, lo que, en consecuencia, permitirá un análisis detallado del discurso en esta interacción. Para precisar ideas, cuando se hace mención a las interacciones de los sujetos, se está aludiendo, básicamente, al sistema semiótico de significación cultural (Radford, 2006b) que se vive en el aula.

Esta investigación se centra en los gestos de un colectivo en interacción, considerando el objeto matemático función de variable real. El interés en este 
contenido se justifica en su carácter de basal para los cursos de cálculo a nivel universitario, tanto dentro de los planes de estudio de las carreras de pedagogía y licenciatura en matemática, como de los de ingeniería. Amparado en ello, se pretende abordar el objeto declarado en un estudio desde el punto de vista gestual, enfatizando en su comportamiento gráfico, y enmarcado en el contexto universitario chileno.

Diversos estudios, en cuanto a la gesticulación, han sido realizados por Radford (2006b) sobre el álgebra en primaria. Sin embargo, no se han reportado muchos estudios de este tipo en la educación superior, a excepción de Arzarello et al. (2009), quienes dan un sentido multimodal en el trabajo de los estudiantes con derivadas y antiderivadas, donde el gesto es parte de dicha multimodalidad. Es por ello que este estudio se considera relevante y novedoso, ya que aborda una temática poco común en el aprendizaje de los estudiantes universitarios, la cual es a través de la interacción con el colectivo por medio de la actividad gestual, cuyos insumos se considerarían un avance importante en la comprensión del objeto función. Por tanto, se espera que, de acuerdo a este enfoque, tales conocimientos sean perdurables en el tiempo.

Los antecedentes mencionados, permiten modificar una clase tradicional de cálculo al momento de tratar las gráficas de funciones de variable real, razón por la cual se parte del supuesto que, por medio de las interacciones señaladas, ello posibilitaría la emergencia de propiedades y/o conceptos a través de la acción, en una actividad que busca evidenciar un cambio de discurso, por medio de realizaciones principalmente gestuales, cuando los estudiantes trabajan en forma colectiva. Con esto se pretende que el estudiante tenga un conocimiento al cual pueda acceder en los cursos posteriores, evocando situaciones que le fueron significativas a través de la acción corporal.

\subsection{Preguntas y OBJetivos DE INVESTIGACIÓN}

De acuerdo a los antecedentes y a los intereses de este estudio, se han generado las siguientes preguntas de investigación: ¿̇uál es el rol del gesto en el discurso matemático de los estudiantes en una actividad con gráficas de funciones en la educación superior?, ¿cómo se evidencia el cambio de discurso matemático en los estudiantes a través de una actividad gestual con gráficas de funciones? Para su concreción, se han planteado los siguientes objetivos: a) 
identificar el rol del gesto en el discurso matemático de los estudiantes de educación superior; b) identificar los elementos del discurso matemático que promueven su cambio en los estudiantes.

\section{MARCO CONCEPTUAL}

Para llevar a cabo esta investigación, se ha recurrido a dos referentes teóricos que se complementan por tener un enfoque no-cognitivista: la Teoría de la Objetivación (Radford, 2006b) y la Teoría Comognitiva (Sfard, 2008). Ambas se centran, por una parte, en el lenguaje y en la interacción del colectivo (como se ha abordado en los trabajos de Vygotsky, 1934/1986); y por otra, en los significados de las palabras (reportado en la obra de Wittgenstein, 1921/2001).

\subsection{TEORÍA DE LA OBJETIVACIÓN}

La forma en que se analiza la interacción de los estudiantes en torno al objeto matemático función de variable real, es a través de lo que Radford (2006b) denomina Teoría de la Objetivación, cuyo foco se encuentra en el aprendizaje, y sobre el cual este autor señala que:

No consiste en construir o reconstruir un conocimiento. Se trata de dotar de sentido a los objetos conceptuales que encuentra el alumno en su cultura. De este modo, la adquisición del saber es un proceso de elaboración activa de significados. (Radford, 2006b, p. 113)

El hecho de dar sentido a los objetos conceptuales, va también de la mano con lo que el mismo autor plantea con respecto al medio, indicando que el pensamiento es una re-flexión mediatizada del mundo, acorde a la forma de la actividad de los sujetos, ligada a lo histórico y a lo cultural (Radford, 2006b). Esta mediación está asociada a lo que Radford (2007) Ilama una superestructura simbólica, que es lo que conforma el sistema semiótico de significación cultural, y está dada, tanto por signos y artefactos, como por una reflexión en torno a ellos. Dicha reflexión, para Radford (2006b), es considerada como pensamiento, $e$ indica que es una movilización dialéctica entre una realidad constituida histórica y culturalmente, y que es modificada por las subjetividades del individuo. 
En síntesis, este primer referente del marco conceptual permitirá analizar la interacción entre los sujetos que están inmersos en la tarea mediante el signo gestual, evocando nociones que, probablemente, tienen de las gráficas de las funciones que han sido construidas por la cultura. Es de interés en este estudio identificar el rol del gesto en el discurso matemático de los estudiantes de educación superior, es decir, entender cómo es que el gesto le permite al estudiante comprender el comportamiento de una gráfica, o lo que caracteriza a una de ellas, y, por consiguiente, el efecto que produce en él abordar un objeto matemático de una manera distinta al trabajo tradicional con lápiz y papel. De este modo, el sistema semiótico de significación cultural será uno de los elementos importantes en el análisis de los datos recopilados en este estudio.

\subsection{TEORÍA COMOGNITIVA}

El segundo referente considerado para este estudio es la Teoría Comognitiva (Sfard, 2008), cuyo foco se encuentra en el aprendizaje basado en las producciones de los estudiantes, las cuales son llamadas por su autora como realizaciones. Con este referente teórico, se pretenden identificar los elementos del discurso matemático que promueven su cambio en los estudiantes, a través de la caracterización del discurso que se da mediante esa gesticulación. Tal discurso, considerado como la unidad de análisis dentro de esta teoría, está caracterizado por una serie de elementos que son reconocidos como recursos y productos para el discurso, los cuales están basados en la comunicación, entendida como:

La actividad que cuenta con una forma regular o inteligible de ser realizada colectivamente, en donde la acción $A$ de un individuo es seguida por la acción $B$ de otro individuo, tal que: (1) A pertenece a un cierto repertorio bien definido de acciones conocidas como comunicacionales, y (2) la acción $B$ pertenece a un repertorio de reacciones que coinciden con $A$, esto es, acciones recurrentemente observadas en conjunción con A. (Sfard, 2008, p. 296)

Teniendo como base la comunicación, es posible definir un discurso en términos de lo planteado por esta teoría, como "Ios diferentes tipos de comunicación, o bien de comognición, que reúna a algunos individuos y excluya a otros" (Sfard, 2008, p. 91). El término comognición proviene de la conjunción de dos 
procesos: el de comunicación y el de cognición, y es lo que da nombre a esta teoría (Sfard, 2008).

Las realizaciones a las que se hizo mención anteriormente, se clasifican de acuerdo a la forma en que se presentan en el discurso matemático, tal como se aprecia en la figura 1 .

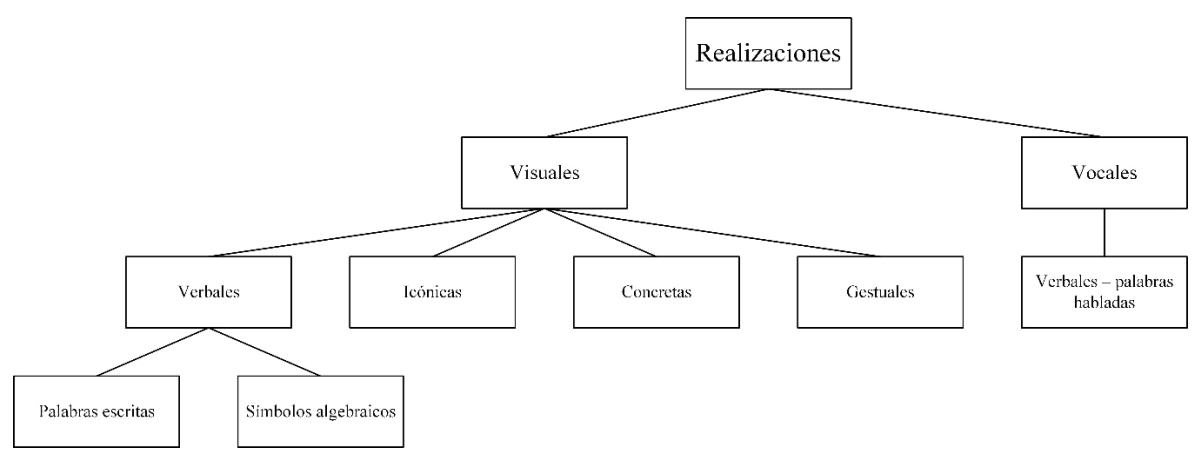

Figura 1. Diferentes tipos (modalidades) de realizaciones en el discurso matemático. Adaptado y traducido desde Sfard (2008, p. 155).

Se destaca el hecho que, lo concreto y lo gestual, trasciende de lo puramente visual-semiótico, pues es también enactivo-kinestésico. Esta idea se basa tanto en los estudios de Varela et al. (1991) como de Soto-Andrade (2018) y, en términos de este estudio, el factor enactivo-kinestésico se considera como un complemento a las realizaciones señaladas por Sfard (2008), quien sólo hace énfasis en lo netamente visual-semiótico.

En cuanto a los elementos que conforman al discurso matemático, se encuentran los recursos (palabras claves y mediadores) y los productos (rutinas y narrativas validadas), los que se describen a continuación, en términos de Sfard (2008):

- Palabras claves: una de las características distintivas del discurso matemático son las palabras claves que éste usa, y que dicen relación con lo que el sujeto ve en el mundo.

- Mediadores visuales: corresponden a objetos visibles que son operados como parte del proceso de comunicación. Los discursos científicos y matemáticos involucran a menudo artefactos simbólicos, creados 
especialmente para un tipo de comunicación particular, como las notaciones matemáticas.

- Narrativas: son cualquier secuencia de expresiones que describe objetos, relaciones entre ellos, o de procesos con o por objetos, sujetos a aprobación o rechazo a través de fundamentos específicos del discurso. Las narrativas validadas, generalmente, se etiquetan de verdaderas. Los criterios de validación pueden variar de un discurso a otro. En matemática, las narrativas validadas por consenso, son aquellas que se conocen por construcciones discursivas, como definiciones, demostraciones y teoremas.

- Rutinas: son patrones característicos del discurso dado, que corresponden a regularidades matemáticas, las cuales son observadas a través de las palabras claves o de los mediadores, para crear y corroborar narrativas numéricas o formas geométricas.

Sfard (2008) establece que no existen fronteras entre los elementos recién mencionados, por lo que, la aparición de cualquiera de éstos, ya es condición para hablar de discurso, tanto del tipo inter-como intrapersonal. No obstante, dependiendo de la comunidad, es que se hacen presentes una o más de estas características del discurso. A modo de ejemplo, en una comunidad de físicos se pueden evidenciar ciertas características, que no estarán presentes en una de matemáticos o de músicos, pues cada colectivo tiene recursos y productos que les son propios como comunidad. En matemática es posible, por ejemplo, que uno de los productos del discurso sea una demostración para validar cierta aseveración dada por una comunidad de matemáticos; en otras disciplinas, por el contrario, bastará con una experimentación concreta, lo que podría ser una validación suficiente para dicha comunidad.

Para efectos de este estudio, el análisis comognitivo entregará pistas para acceder tanto a los recursos como a los productos de los discursos y, de esta forma, se pretende evidenciar que la gesticulación es el motor del desarrollo del discurso matemático del estudiante. El hecho de abordar la Teoría Comognitiva (Sfard, 2008) y la Teoría de la Objetivación (Radford 2006b) en este trabajo, radica en que tienen como elemento en común las realizaciones a las que alude Sfard (2008) y que, en palabras de Radford (2006b, 2007), se refieren a un signo, el que, para este caso, es una acción corporal llamada gesto. Ahora bien, la diferencia entre ambos referentes es, primeramente, la especificidad que la Teoría Comognitiva entrega en cuanto a los signos, entendidos como realizaciones para Sfard (2008), lo que hace más detallado el análisis didáctico, 
permitiendo describir de forma amplia el sistema semiótico de significación cultural, del cual habla Radford (2006b), en que conviven e interpretan los individuos en el mundo. En este sentido, se habla del mundo en el cual los estudiantes conviven con la matemática en la práctica habitual, pero ahora, a través de los gestos. Siendo así, la Teoría Comognitiva da un sustento a aquel sistema semiótico de significación cultural de manera más sólida, dejando en evidencia los elementos del discurso como signo específico, lo que a su vez permite un análisis mucho más detallado de la actividad realizada por los individuos.

\section{METODOLOGÍA}

Este estudio se inscribe bajo un paradigma cualitativo, y se ha empleado la metodología del Estudio de Casos (Stake, 1995), pues interesa, en primer lugar, identificar el rol del gesto en el discurso matemático de los estudiantes de educación superior, para así poder identificar los elementos de este discurso que promueven su cambio en los estudiantes. Desde la perspectiva matemática, el comportamiento de las funciones y sus propiedades son analizados bajo la mirada del cálculo diferencial, mientras que el cálculo de áreas entre curvas, es abordado por el cálculo integral. Para este último, se requiere de un conocimiento adecuado, tanto de las gráficas de las funciones, indicando cortes con los ejes e intersecciones de las curvas involucradas, como de sus propiedades, las que son imprescindibles para el estudio de las series. Por ello es que, para esta propuesta, se utilizan funciones habituales dentro de los cursos de cálculo diferencial e integral de una variable, así como en los de precálculo o de fundamentos de la matemática, a saber, funciones polinómicas, trigonométricas, entre otras.

\subsection{PARTICIPANTES}

Los sujetos a los cuales se aplicó esta propuesta son estudiantes (entre 18 y 21 años de edad) que pertenecen a las carreras de Ingeniería Química Industrial e Ingeniería Bioquímica en una universidad chilena. Los sujetos se encontraban cursando la asignatura de Cálculo I, en un curso conformado por 30 alumnos, de los cuales, 8 participaron voluntariamente de la actividad (rotulados desde E1 hasta E8), organizándose en parejas para desarrollarla. 


\subsection{LA PROPUESTA}

La actividad propuesta consistió en que los estudiantes representaran diversas funciones a través de gestos. El registro en video de la actividad fue realizado por los mismos estudiantes, ello para propiciar una mayor confianza, fluidez y naturalidad en sus acciones. La elección de estas funciones era decisión de cada grupo, aunque se les indicó que fuera lo más variada posible.

La implementación de la actividad se llevó a cabo durante dos sesiones del curso, con una duración de 90 minutos cada una, pero sin la presencia del docente en la sala de clases, ello para contribuir a la espontaneidad de la actividad durante la ejecución de la misma. Además, se les indicó a los estudiantes que podían usar cualquier tipo de objeto concreto si lo consideraban pertinente. Al término de la semana de clases, se pidió a los participantes que entregaran al docente su edición de video.

\subsection{TÉCNICAS Y CATEGORÍAS DE ANÁLISIS}

Como el interés de este estudio se centra en las interpretaciones que realizan los estudiantes en torno a la actividad gestual, se registraron en video sus intervenciones para analizar tanto las gesticulaciones que los sujetos realizan como el diálogo que se genera entre ellos. Por tanto, las unidades de análisis de esta investigación son: el sistema semiótico de significación cultural y el discurso matemático. El primero es entendido, en términos de esta investigación, como la cultura en el aula de matemática bajo la visión del signo gesto, es decir, vivir la matemática con la corporalidad a disposición del individuo que quiere comunicar alguna propiedad y/o conceptualización del objeto matemático en cuestión. El segundo, por su parte, es entendido en este estudio como la comunicación en el colectivo a través de diversas realizaciones que permiten develar la interacción de los individuos (estudiantes) con la matemática (funciones de variable real) a través de lo visual y lo gestual, permitiendo conocer -por parte del investigador- aquellas realizaciones que priman en este interactuar.

Para analizar los resultados, se han propuesto las siguientes categorías de análisis:

- Una primera categoría centrada en el sistema semiótico de significación cultural, en que se evalúa el trabajo en el colectivo bajo la interpretación 
de los investigadores, analizando si estas parejas de estudiantes dan sentido a los objetos abstractos, más en concreto, cómo las parejas son capaces de mostrar una propiedad, concepto o comportamiento de una gráfica.

- Una segunda categoría centrada en el discurso matemático, específicamente, en el tipo de realizaciones (ver figura 1) que privilegian los estudiantes para dar significado matemático y comunicar dicho significado al otro, permitiendo así discriminar entre una realización y otra.

\section{PRESENTACIÓN Y ANÁLISIS DE DATOS}

En este apartado se analiza la actividad didáctica implementada, presentando, además, algunas capturas de video que se consideran como relevantes, pues evidencian el trabajo colectivo y la comunicación que se establece entre los estudiantes. De estas producciones se caracterizan las realizaciones de los sujetos, las que son posibles evidenciar tanto en forma visual como vocal. Sobre esto último, es posible evidenciar las realizaciones vocales a través de la transcripción de algunos diálogos relevantes, donde se indican entre corchetes [...] las acciones corporales de los sujetos, y en cursivas lo que enuncian verbalmente.

En el caso de las estudiantes E1 y E2, la forma de dar información sobre la gráfica de funciones, fue a través de una realización visual y vocal, caracterizada por gestos y palabras habladas. Dentro de las realizaciones visuales, se destacan las realizaciones verbales escritas por medio de los símbolos algebraicos, los que fueron escritos como una lista en la pizarra con anterioridad, que incluye funciones polinomiales, trigonométricas y exponenciales. La estudiante E1 es quien gesticula, y procede con una realización icónica para mostrar a E2 la gráfica a la que se está refiriendo. En esta lista -considerada como una realización simbólica algebraica- se encuentra la función a la que E1 se refiere con el movimiento de sus brazos (ver figura 2). El siguiente diálogo se desarrolló entre las estudiantes:

E1: [alza los brazos, cada uno formando una especie de recta inclinada].

E2: iAh!, la función valor absoluto.

E1: [marca en el listado de la pizarra, la función que indicó E2, $y=|x|$ ]. 


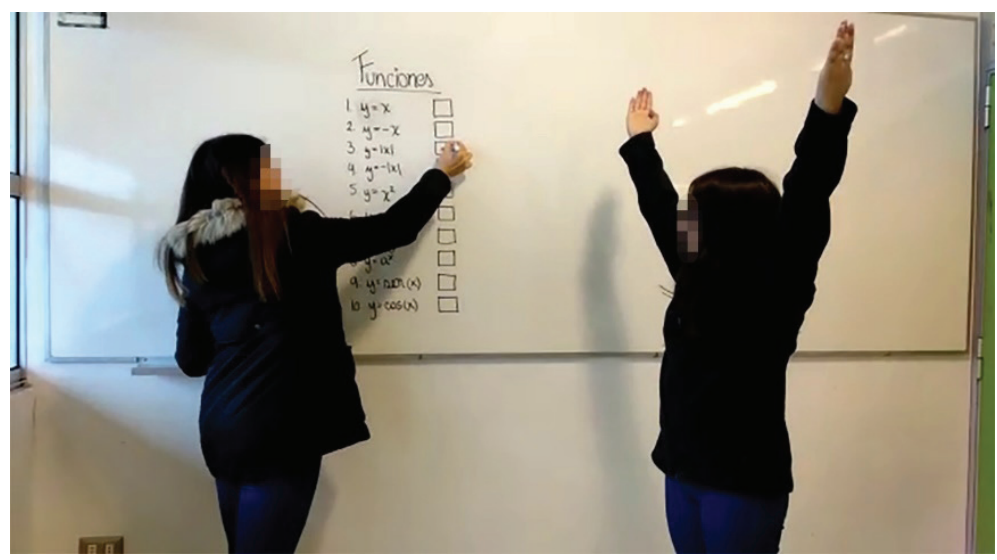

Figura 2. Realización de E1 y E2.

En el caso de los estudiantes E3 y E4, el registro en video evidencia realizaciones visuales y también vocales. E3 es quien gesticula y E4 dice oralmente a qué función corresponde. Las realizaciones visuales se inclinan hacia la evocación de lo icónico por medio de E3, quien muestra de forma corporizada la gráfica de la función que desea que identifique E4. En un momento dado, E3 describe una función que no es visualizada de forma clara por E4. A pesar de que E4 no hace declaraciones con respecto a alguna función, su silencio ante las gesticulaciones de E3 hace que este último detalle en ella, dando más información para que sus gestos sean comprendidos por E4. La información adicional está relacionada con datos que involucran un sistema de referencia, como se detalla a continuación:

E3: [muestra el sistema de ejes $X Y$, de manera corporizada, indicando con los dedos dichos ejes, y mostrando que parte desde el origen].

E3: [procede con sus brazos a gestualizar el movimiento que describe la curva de la función, indicando que esta se mueve desde el origen hacia la derecha del sistema de coordenadas $X Y$ ].

E4: YYa sé!, la función raíz cuadrada.

A diferencia del grupo formado por E1 y E2, los gestos realizados por E3 y E4, son llevados a cabo de manera más activa, pues estos estudiantes evidenciaron un discurso matemático más evolucionado, el cual muestra más elementos de la matemática que son corporizados, como los ejes $X$ e $Y$, y el origen (o punto 
de referencia) a partir de dichos ejes, por lo que, de esta forma, se promueve aún más el movimiento del cuerpo para describir el objeto. Es así como se puede evidenciar que E3 se indica a sí mismo (ver figura 3, izquierda) para señalar desde dónde parte la gráfica de la función, y qué movimiento describe (ver figura 3, derecha), a saber, su brazo extendido indica el eje $X$, lo cual se hace evidente para E4 quien, de acuerdo a lo que muestra el video, se encuentra sentado observando los gestos que produce E3, e indica oralmente que es la función raíz.
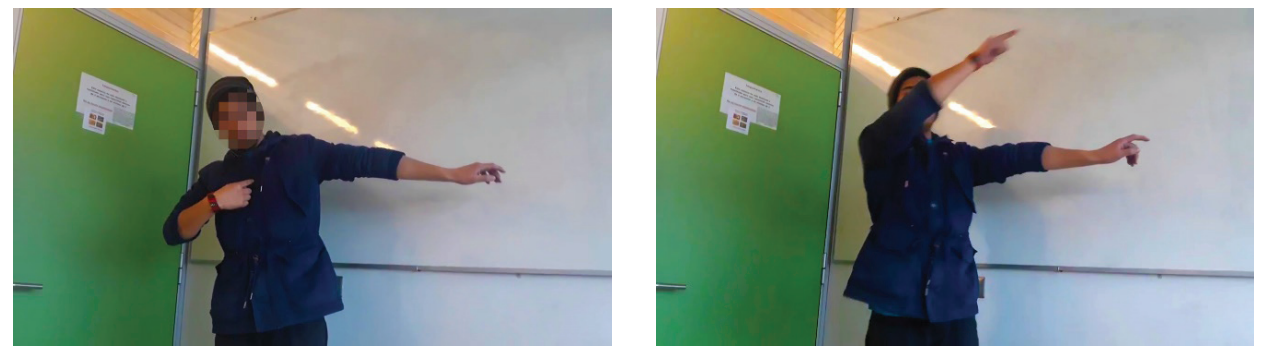

Figura 3. Realización de E3.

En el caso de los estudiantes E5 y E6, se evidencian realizaciones visuales y vocales de funciones trigonométricas. En una primera instancia, el estudiante E5 realiza la gesticulación, mientras que E6 responde oralmente a qué función se refiere E5:

E5: [gesticula con sus brazos una función en forma de onda].

E6: Es la función coseno.

E5: [indica con la cabeza que no].

E6: Es el seno.

Se debe destacar la importancia del cuerpo de E5 (véase figura 4) para que E6 diga que es la función seno, pues la cabeza de E5 toma el rol de ser el eje $Y$, mientras que los brazos corresponden a las ramas de la función en cuestión. Tal vez, esa situación confundió a E6 entre las funciones coseno y seno, para decir finalmente que esta última correspondía a la gesticulada por E5, lo cual nos sugiere que el sistema de ejes cartesianos se torna relevante en la representación de la función en cuestión. 


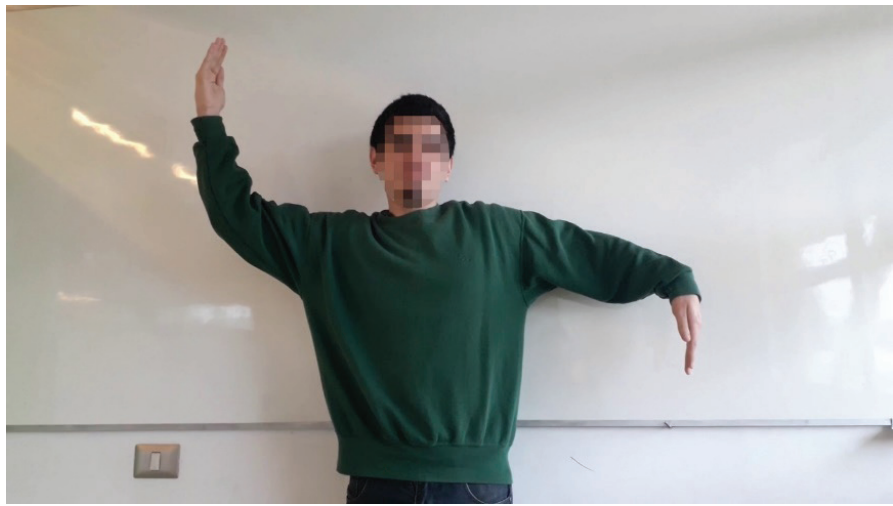

Figura 4. Realización de E5.

En una segunda instancia, se invierten los roles y es el turno de la estudiante E6 en mostrar su gesticulación (véase figura 5):

E5: Es el seno... ino, perdón!, es el coseno.

Nuevamente, el eje $Y$ es el elemento que dirige la respuesta a la gesticulación realizada. Es destacable el hecho de que, tanto en las realizaciones de E3 y E4 como de E5 y E6, los ejes coordenados toman gran importancia para quien visualiza la gesticulación.

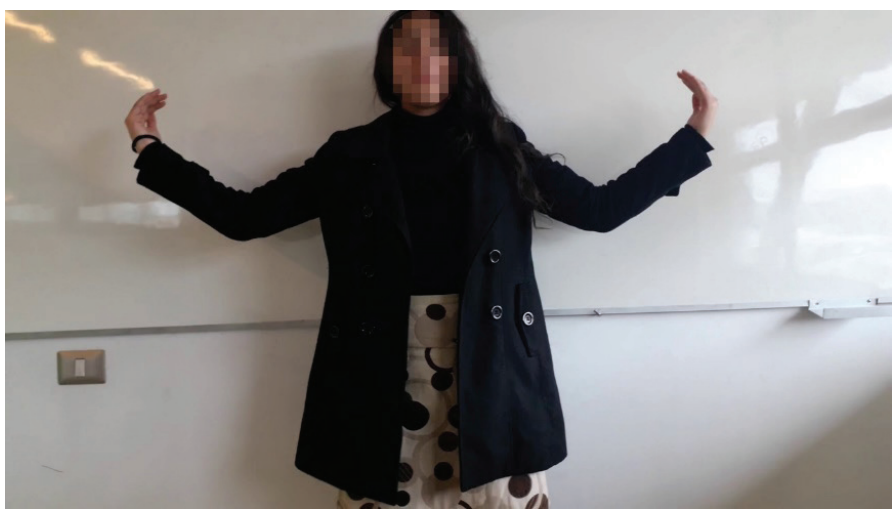

Figura 5. Realización de E6. 
En el siguiente caso, se destaca una realización visual y vocal, con palabras que van describiendo la gesticulación de una función. La estudiante E7 está atenta a las descripciones que hace E8, quien también indica el orden de las palabras que se necesitan para la descripción de la función. La siguiente transcripción de diálogo describe la situación entre ambos estudiantes:

E8: [mueve sus brazos y manos en forma de ondas, repitiendo la acción hacia los lados].

E7: Eh, función trigonométrica.

E8: [continúa moviendo los brazos hacia los lados, espera algo más de E7].

E7: Se repite... periódica.

E8: [levanta sus dedos pulgares, indicando que es correcto].

E8: [levanta el dedo índice].

E7: Ah, primera palabra.

E8: [desliza su mano indicando una inclinación, de arriba hacia abajo] (véase figura 6, izquierda).

E7: Ya... intersección.

E8: [levanta el dedo índice, apuntando al eje $Y$ ] (véase figura 6, centro) [indica con sus dedos índice y medio, tanto la segunda palabra como el corte en forma $Y$ ] (véase figura 6, derecha).

E7: Ah, corta al eje $Y$ en 1.

E8: [levanta sus dedos pulgares, indicando que es correcto].

E7: iSeno!

E8: [mueve sus dedos índices negativamente].

E7: Entonces, es coseno.

E8: [levanta sus dedos pulgares, indicando que es correcto].
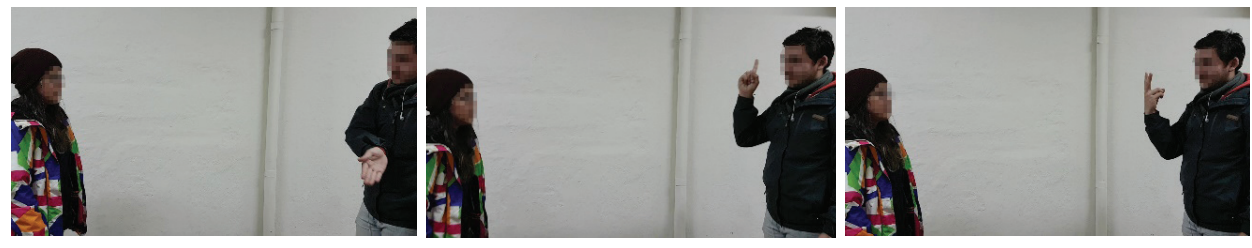

Figura 6. Realización de E7 y E8. 
Se observa en la secuencia de gestos y en el diálogo de los estudiantes E7 y E8, que la relevancia, además de los gestos, está en la palabra, cuya significación es propia del mundo de la matemática. Es decir, se genera un discurso matemático entre E7 y E8, que trae consigo palabras claves, como el término 'intersección con los ejes', acompañado del movimiento que describe la función en forma repetida, y que permite la emergencia de la palabra clave 'periódica'. Esta mezcla adecuada entre palabras y gestos, permitió también rescatar propiedades de las funciones trigonométricas, como lo es en el caso de $\operatorname{sen}(x)$, en la que es posible identificar una intersección con el eje $Y$ en el punto $(0,1)$, cuestión que es evidenciable cuando E8 indica que hubo un error por parte de E7, al declarar inicialmente que la función era $\cos (x)$.

\section{DISCUSIÓN Y CONCLUSIONES}

De acuerdo a los resultados, se observa que las parejas en su mayoría responden de acuerdo a la necesidad de comunicación a través del signo gesto, conformando de este modo un espacio común de interacción en el aula y con la matemática, dando significado de forma colectiva y aplicando la matemática en el acto. Sobre el discurso, en las realizaciones de los grupos conformados por E3-E4, E5-E6 y E7-E8, no se privilegia la simbología algebraica escrita, lo que evidencia que las realizaciones de los estudiantes fueron principalmente visuales, del tipo gestuales. Ello sugiere que lo simbólico algebraico puede ser un factor prescindible para describir un objeto matemático. En cambio, para el grupo de las estudiantes E1-E2, la lista de funciones cobró un carácter más relevante para el desarrollo de la actividad.

Teniendo en consideración la investigación de McNeill (1992) sobre las gesticulaciones, se destaca el rol de los dedos en la actividad desarrollada por los estudiantes E7 y E8, cuando indican la cantidad de intersecciones de una función con los ejes, y la de los estudiantes E3 y E4, para indicar el punto de referencia u origen de la función raíz.

El análisis de la implementación de este estudio, mostró las diversas maneras de llevar a cabo la actividad dada a los estudiantes. Por una parte, grupos como E1-E2, se apoyaron en realizaciones simbólicas algebraicas, las cuales hacen notar la dependencia del recurso algebraico para reafirmar sus conocimientos asociados con la actividad gestual. Por el contrario, los demás grupos no consideran el apoyo de la simbología algebraica, centrándose en los 
movimientos corporales y acentuando aún más sus gesticulaciones para la evolución del discurso en su par, lo cual evidencia un cambio de discurso in situ.

La obtención de palabras claves fue uno de los recursos que permitieron continuar con el discurso de E7 y E8, las que responden a los fenómenos gesticulados. Es así que se pone de relieve el significado de la palabra del cual habla Wittgenstein (1921/2001), quien alude a aspectos que trascienden la matemática formal y que tienen, por una parte, relación con una actividad contextualizada en torno a este tipo de fenómenos y, por otra, aún más significado matemático para el colectivo en su necesidad de comunicar propiedades de acuerdo al contexto en el cual viven la matemática. Ejemplo de ello es cuando E8 gesticulaba una acción repetidamente para dar a entender un concepto en la función, lo que induce la aparición de la palabra clave 'periodicidad', como una particularidad de esta función. Sin embargo, en las realizaciones de los otros grupos de estudiantes, no se evidenció un gesto que involucrara esta palabra clave, situación que pone de manifiesto un interés y una preocupación de la dupla E7-E8 por hacer emerger otras propiedades de la función en cuestión como una característica particular. Además de ello, este grupo demostró, a través de lo gestual, que la función $\operatorname{sen}(x)$ es entendida como un objeto matemático que presenta otras características relevantes además de su forma, como lo es la intersección con el eje $Y$ en el punto $(0,1)$.

En cuanto a los objetivos de esta investigación, es posible decir que el rol del gesto, en términos de lo que declara Sfard (2008), constituye un mediador ad hoc que incentiva la comunicación matemática de manera más natural en el colectivo de los estudiantes. Así lo demostró la actividad implementada, en la que los individuos se sintieron motivados a comunicarse entre ellos a través de diversas realizaciones, y si bien la naturaleza de la propuesta permitió que se llevara a cabo de manera grata, ello no significó perder de vista el objeto matemático en estudio: las funciones de variable real. De este modo, los gestos conforman un mediador que permite el enunciado de propiedades y/o características de las funciones. Esto refleja la acción del gesto como un motor desarrollador de un discurso en el individuo que visualiza y escucha lo que realiza el otro.

Una de las observaciones interesantes con respecto a los gestos es que, si bien en los comienzos no son de carácter matemático, con el avance de la actividad empieza a tomar un tono más formal. Esto se genera debido a que, conforme se entrega nueva información, las gesticulaciones se tornan más complejas, y deben ser interpretadas por otro sujeto, lo que le obliga -en cierto 
modo- a recurrir a parte de su conocimiento que le evoque un lenguaje adecuado, acorde a lo gesticulación que observa.

Debido a que en esta investigación se pretendió identificar los elementos del discurso matemático que promueven su cambio en los estudiantes, es decir, su evolución, se constató que esto fue posible gracias a las realizaciones, tanto visuales como vocales, que fueron evidenciadas a medida que avanzaba el gesticulante en su tarea de comunicar, para que con los gestos se develaran en otro individuo las propiedades y características del objeto matemático de interés en esta investigación, permitiendo evidenciar un discurso predominante en palabras claves y narrativas validadas mediante los gestos.

Un elemento de relevancia en esta investigación fue el sistema semiótico de significación cultural en la actividad de los sujetos que, de acuerdo a los resultados de las experiencias realizadas, reafirman la idea de Radford (2006b) sobre los efectos en el pensamiento del sujeto, ya que dicho sistema, a través del medio y sus herramientas, implica una realidad que se constituye de manera histórica y cultural, la que el individuo refracta y modifica según sus subjetividades. Así, la realidad presentada a través de los gestos, es una señal que el individuo interpreta y modifica de acuerdo a su experiencia escolar, la cual es ampliada a través de este enfoque de carácter gestual, que hemos denominado como el motor del desarrollo del discurso.

Este motor del desarrollo del discurso, a saber, los gestos, se plantea como una manera de transitar desde lo que alude Sfard (2008) como las ritualidades, a las exploraciones, ello para dar sentido al quehacer matemático en el aula. En otros términos, se plantea la idea de pasar de un espacio basado en actividades dadas por reglas matemáticas, sólo por pertenecer a un grupo que realiza tales prácticas, tal vez sin entender en profundidad, a un espacio basado en experiencias ricas con el colectivo, las cuales darían sentido y vida al objeto matemático en cuestión. Siguiendo esta idea, compartimos también la nueva visión de la rutina a la que se refieren Lavie, Stein y Sfard (2019), ya que ha sido posible constatar que estas evolucionan en cada grupo de estudiantes, permitiendo así pasar a la exploración, y dejando de lado esta visión de rutina meramente como un patrón invariante.

La concepción del 'ser con el otro', que plantea Radford (2006b), es una visión que entendemos como la mejora en el aprendizaje de los alumnos en su búsqueda de dar explicación a aquellos objetos tan abstractos como los matemáticos, pero en este caso, con un enfoque centrado en lo enactivo-kinestésico, lo que permite generar otro tipo de explicaciones para el objeto matemático 
funciones. Así, la gestualidad promovería conocimiento matemático de manera más dinámica, y con ello una reflexión profunda del estudiante con el objeto en cuestión, generando palabras más especializadas en el ámbito matemático y, junto a ello, propiedades que emergen a raíz de esta actividad. Entonces, ¿̇cuál sería el beneficio de este tipo de actividades en el aula? Uno de los beneficios que traerían consigo las actividades gestuales, es la perdurabilidad en el tiempo del conocimiento adquirido en estas, para así, establecer una base de conocimientos disponible para cursos posteriores al cálculo fundamental o cursos equivalentes. En esta misma línea, se propone realizar estas actividades incluso desde los niveles iniciales en la formación de los estudiantes, más allá que la línea del álgebra temprana, como se ha mencionado anteriormente. En este sentido, el discurso matemático, por parte de los estudiantes, se vuelve más rico y accesible en un determinado momento.

Una proyección de este estudio sería la instauración de diversos sistemas de signos que enriquecerían el discurso matemático de los estudiantes en el aula. De acuerdo a esto último, se pretenden explorar las propiedades de objetos matemáticos más complejos como, por ejemplo, las derivadas de funciones, indicando con los dedos los máximos y los mínimos, cómo varían las pendientes de las rectas tangentes a ciertas curvas, etcétera.

\section{AGRADECIMIENTOS}

Este estudio fue realizado en el marco del Proyecto ANID/PFCHA nro. 21180980 (Chile) 


\section{REFERENCIAS}

Alibali, M. W., Nathan, M. J., Boncoddo, R. y Pier, E. (2019). Managing common ground in the classroom: teachers use gestures to support students' contributions to classroom discourse. ZDM Mathematics Education, 51(2), 347-360. https://doi.org/10.1007/ s11858-019-01043-x

Artigue, M. (2011). Tecnología y enseñanza de las matemáticas: desarrollo y aportes de la aproximación instrumental. Cuadernos de Investigación y Formación en Educación Matemática, 6(8), 13-33.

Arzarello, F., Paola, D., Robutti, O. y Sabena, C. (2009). Gestures as semiotic resources in the mathematics classroom. Educational Studies in Mathematics, 70(2), 97-109. https://doi.org/10.1007/s10649-008-9163-z

Gamboa, R. (2007). Uso de la tecnología en la enseñanza de las matemáticas. Cuadernos de Investigación y Formación en Educación Matemática, 2(3), 11-44.

Goldin-Meadow, S. (2003). Hearing gesture: How our hands help us think. Cambridge, MA: Harvard University Press.

Gómez-Chacón, I. M. (2010). Actitudes de los estudiantes en el aprendizaje de la matemática con tecnología. Enseñanza de las Ciencias, 28(2), 227-244. https://doi. org/10.5565/rev/ec/v28n2.197

Kita, S. (2000). How representational gestures help speaking. En D. McNeill (Ed.), Language and Gesture (pp. 162-185). Cambridge University Press. https://doi.org/10.1017/ CB09780511620850.011

Lavie, I., Steiner, A. y Sfard, A. (2019). Routines we live by: from ritual to exploration. Educational Studies in Mathematics, 101(2), 153-176. https://doi.org/10.1007/s10649018-9817-4

Mancilla, M. y Puentes, C. (2015). La gestualidad y los segmentos narrativos propuestos por Labov: análisis de una narración. Lengua y Habla, 19, 216-230.

McNeill, D. (1992). Hand and Mind: What gestures reveal about thought. University of Chicago Press.

McNeill, D. (2005). Gesture and Thought. University of Chicago Press.

Radford, L. (2006a). Introducción: Semiótica y Educación Matemática. RELIME: Revista Latinoamericana de Investigación en Matemática Educativa, 9(Número especial), 7-21.

Radford, L. (2006b). Elementos de una teoría cultural de la objetivación. RELIME: Revista Latinoamericana de Investigación en Matemática Educativa, 9(Número especial), 103-129.

Radford, L. (2007). Semiótica cultural y cognición. En R. Cantoral, O. Covián, R. Farfán, J. Lezama y A. Romo (Eds.), Investigaciones sobre enseñanza y aprendizaje de las 
matemáticas: Un reporte Iberoamericano (pp. 669-689). Díaz de Santos-Comité Latinoamericano de Matemática Educativa A.C.

Radford, L. (2010). Signs, gestures, meanings: Algebraic thinking from a cultural semiotic perspective. En V. Durand-Guerrier, S. Soury-Lavergne y F. Arzarello (Eds.), Proceedings of the Sixth Congress of the European Society for Research in Mathematics Education (pp. XXXIII-LIII). Institut National de Recherche Pédagogique, ERME.

Radford, L., Edwards, L. y Arzarello, F. (2009). Introduction: Beyond words. Educational Studies in Mathematics, 70(2), 91-95. https://doi.org/10.1007/s10649-008-9172-y

Sfard, A. (2008). Thinking as Communicating: Human Development, the Growth of Discourses, and Mathematizing. Cambridge University Press.

Sfard, A. y McClain, K. (2002). Analyzing tools: Perspectives on the role of designed artifacts in mathematics learning. The Journal of the Learning Sciences, 11(2-3), 153161. https://doi.org/10.1080/10508406.2002.9672135

Soto-Andrade, J. (2018). Enactive metaphorising in the learning of mathematics. En G. Kaiser et al. (Eds.), Invited Lectures from the 13th International Congress on Mathematical Education (pp. 619-637). Springer. https://doi.org/10.1007/978-3-319-72170-5_34

Stake, R. E. (1995). The Art of Case Study Research. SAGE.

Suárez, F. y Pietrosemoli, L. (2007). Gestualidad y estructura narrativa. Análisis de un caso. Lengua y Habla, 11, 79-90.

Varela, F. J., Thompson, E. y Rosch, E. (1991). The Embodied Mind: Cognitive Science and Human Experience. MIT Press.

Vygotsky, L. S. (1986). Thought and Language (A. Kozulin, Trad.) (2da ed.). The MIT Press. (Trabajo original publicado en 1934).

Wittgenstein, L. (2001). Tractatus Logico-Philosophicus (D. F. Pears y B. F. McGuinness, Trads.) (2da ed.). Routledge. https://doi.org/10.4324/9780203010341 (Trabajo original publicado en 1921)

LUIS SANDOVAL-TRONCOSO

Dirección postal: Departamento de Matemática y Estadística, Facultad de Ingeniería y Ciencias, Universidad de la Frontera. Calle Francisco Salazar 01145, Temuco, Chile

Teléfono: $\quad$ +56942312256 